\title{
Methods to Recruit Hard-to-Reach Groups: Comparing Two Chain Referral Sampling Methods of Recruiting Injecting Drug Users Across Nine Studies in Russia and Estonia
}

\author{
Lucy Platt, Martin Wall, Tim Rhodes, Ali Judd, \\ Matthew Hickman, Lisa G. Johnston, Adrian Renton, \\ Natalia Bobrova, and Anya Sarang
}

\begin{abstract}
Evidence suggests rapid diffusion of injecting drug use and associated outbreaks of HIV among injecting drug users (IDUs) in the Russian Federation and Eastern Europe. There remains a need for research among non-treatment and community-recruited samples of IDUs to better estimate the dynamics of HIV transmission and to improve treatment and health services access. We compare two sampling methodologies "respondent-driven sampling" (RDS) and chain referral sampling using "indigenous field workers" (IFS) to investigate the relative effectiveness of RDS to reach more marginal and hard-to-reach groups and perhaps to include those with the riskiest behaviour around HIV transmission. We evaluate the relative efficiency of RDS to recruit a lower cost sample in comparison to IFS. We also provide a theoretical comparison of the two approaches. We draw upon nine community-recruited surveys of IDUs undertaken in the Russian Federation and Estonia between 2001 and 2005 that used either IFS or RDS. Sampling effects on the demographic composition and injecting risk behaviours of the samples generated are compared using multivariate analysis. Our findings suggest that RDS does not appear to recruit more marginalised sections of the IDU community nor those engaging in riskier injecting behaviours in comparison with IFS. RDS appears to have practical advantages over IFS in the implementation of fieldwork in terms of greater recruitment efficiency and safety of field workers, but at a greater cost. Further research is needed to assess how the practicalities of implementing RDS in the field compromises the requirements mandated by the theoretical guidelines of $R D S$ for adjusting the sample estimates to obtain estimates of the wider IDU population.
\end{abstract}

KEYWORDS Estonia, HIV, Indigenous field workers, Injecting drug users, Respondentdriven sampling, Russia.

Platt, Rhodes, Hickman, and Bobrova are with the Centre for Research on Drugs and Health Behaviour, Department of Public Health and Policy, London School of Hygiene and Tropical Medicine, London, UK; Wall and Renton are with the Institute for Health and Human Development, University of East London, London, UK; Judd is an independent consultant with the Medical Research Council Clinical Trials Unit, London, UK; Hickman is with the Department of Social Medicine, University of Bristol, Bristol, UK; Johnston is an independent consultant with Institute for Global Health, University of California, San Francisco, CA, USA; Sarang is an independent consultant with the Central and Eastern European Harm Reduction Network, Vilnius, Lithuania.

Correspondence: Lucy Platt, Centre for Research on Drugs and Health Behaviour, Department of Public Health and Policy, London School of Hygiene and Tropical Medicine, London, UK. (E-mail: lucy.platt@lshtm.ac.uk) 


\section{INTRODUCTION}

Evidence suggests recent diffusion of injecting drug use and associated HIV infection in the Russian Federation since 1996. ${ }^{1,2}$ Approximately 60\% of HIV case reports have been associated with injecting drug use, ${ }^{1,3}$ with recent estimates indicative of increased sexual HIV transmission. ${ }^{4}$ According to UNAIDS classifications, HIV in much of the Russian Federation and former Soviet Union is a concentrated epidemic, with prevalence consistently above $5 \%$ in a single risk group (i.e., IDUs) but less than $1 \%$ in the general population. ${ }^{5}$ Concentrated epidemics require targeted surveillance of the population group most at risk in order to track the spread within that group as well as potential transmission to others.

Surveillance among IDUs is problematic, and there has been much discussion on the merits of different methods to recruit marginalized and hidden groups for these purposes. ${ }^{6-8}$ We know that surveying drug users in treatment settings misses an important segment of the drug using population. Evidence suggests that behaviours, characteristics and HIV prevalence amongst IDUs in treatment often systematically differ to IDUs not in treatment. ${ }^{9-13}$

Many surveillance studies of IDUs conducted in the 1990s relied on nonprobability sampling such as convenience, snowball sampling or chain referral sampling to recruit members of the target group. ${ }^{12,14}$ These methods work on the assumption that peers are better able to recruit members of a hidden population than researchers. ${ }^{15}$ Typically studies employed 'privileged access interviewers' or 'indigenous field workers' to recruit IDUs from community settings. Indigenous field workers are interviewers who are either current or former drug users or individuals who have experience working with drug users and have privileged access to IDU networks. Over the last 15 years, this has become the established sampling method for recruiting hidden populations of IDUs and sex workers both in the UK and internationally. ${ }^{16-21}$

A refinement of the chain referral methodology called respondent-driven sampling (RDS), has recently been developed. ${ }^{22}$ RDS is inspired by the insight of "small world theory" that suggests that every person is indirectly associated with every other person through approximately six intermediaries, ${ }^{23}$ and therefore that everyone in a defined population could be potentially reached through several waves of recruitment in a chain-referral sample. ${ }^{24}$ This implies that there is a probability greater than zero that everyone in that population will be sampled.

The unique selling point of RDS is that the collection of data on participants' social networks allow for adjustment for non-random recruitment. RDS uses social network data to make inferences about the wider target population from which the sample is drawn to provide proportional population estimates of characteristics and behaviours. ${ }^{24,25}$ In this paper, we do not attempt to test the statistical superiority of RDS in providing 'population' estimates over other sampling strategies but instead focus on RDS as a recruitment strategy examining the unadjusted RDS sample characteristics.

This paper compares two sampling methodologies, RDS and chain referral sampling using indigenous field workers (IFS), in terms of cost effectiveness, duration of fieldwork and effects on the demographic composition of the sample. First we offer a theoretical descriptive comparison of the two approaches. 


\section{THEORETICAL COMPARISON OF THE SAMPLING METHODS}

\section{Indigenous Field Worker Sampling}

The IFS recruitment method uses a standard chain referral approach. Indigenous field workers undergo training covering aims of the study, fieldwork protocols, ethics, informed consent, interview skills and safety procedures. Field workers (FWs) identify individuals known to them from IDU networks, recruit; and then interview them in community settings, separate from the rest of the research team. Eligible participants are given an incentive to take part and also asked to introduce their peers to the FW. The use of multiple site and network recruitment ensures a wide coverage of the population, providing as representative a sample as possible. There is some evidence that the use of FWs with direct access to IDU social networks facilitates recruitment and reduces masking (undersampling reclusive respondents), volunteer bias (oversampling cooperative respondents) and underreporting of socially undesirable behaviours. ${ }^{10,26}$

\section{Respondent-driven Sampling}

In RDS, a fixed site or "store front" is established where all interviewing takes place, providing the research team with greater control over the fieldwork. Unlike IFS and other chain referral samples, RDS uses a dual incentive system, a primary incentive for participating in the study and a secondary incentive for recruiting others into the study. ${ }^{22}$ Sampling begins with a set of initial subjects who serve as 'seeds' for an expanding chain of referrals, with respondents from each link in the chain or 'wave' referring respondents who form subsequent waves. Rather than being asked to identify their peers to interviewers, respondents inform their peers about the study and allow them to decide independently whether they want to participate or not. This theoretically reduces masking since recruiters are part of the target group with direct access to other IDUs, and it reduces volunteer bias since recruitees decide themselves whether to participate.

Information on the relationships between recruiters and recruited and their estimated network size is collected during the interview to allow for the calculation of selection probabilities. ${ }^{27}$ This information is used to assess homophily, the extent to which recruiters are likely to recruit individuals similar to themselves, and to weight the sample to compensate or control for differences in network size, homophily and recruitment success. ${ }^{24}$

\section{MATERIALS AND METHODS}

\section{Data Collection}

Between 2001 and 2005, we undertook nine community surveys of IDUs in the Russian Federation and Estonia (Table 1). Four studies used IFS to recruit IDUs, and five used RDS. All IDUs were recruited from community settings. Seven of the studies had an epidemiological focus and measured the prevalence of HIV, HCV and associated injecting and sexual risk behaviours in IDUs. ${ }^{13,28,29}$ Two of the studies collected data on the social and economic characteristics of IDUs. ${ }^{30}$ All studies collected some standardised indicators and defined current IDUs as individuals who injected drugs for non-medical purposes in the last 4 weeks. 


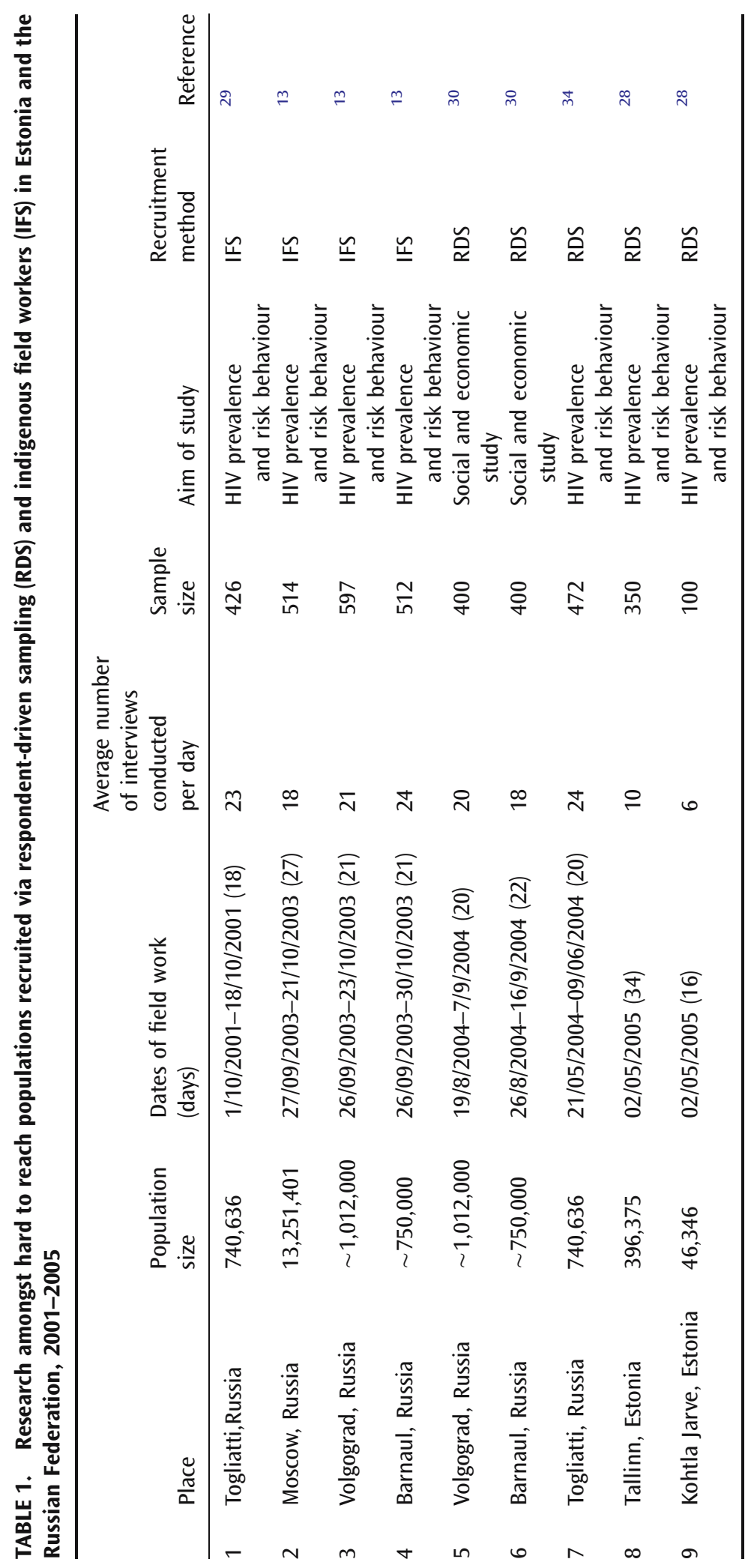


For each of the IFS studies, IDUs were recruited using a team of 10-12 FWs at each site. Settings included street locations and respondents' homes but excluded drug treatment centres and STI clinics. Volunteers and outreach workers at local harm reduction non-governmental organizations (NGOs) were employed as FWs, as well as two researchers at a local university in each site. In all IFS studies, two experienced supervisors from Moscow and a researcher from the UK provided technical expertise and management for all studies. Measures to ensure data quality and to minimise network bias included limiting the number of interviews per FW, random spot-checks in the field, and follow-up validation interviews with $10 \%$ of participants. Primary incentives included HIV prevention materials (including needles/syringes), chocolates and cigarettes.

In each RDS study, recruitment was undertaken by a team of seven to eight FWs at each site. The interview team comprised trained research staff from a local university, two FWs recruited from local harm reduction NGOs, two to three trained research staff from a local university, and two supervisors from Moscow. A researcher from the UK was also present at the studies, with the exception of the two socio-economic studies in Volgograd and Barnaul, Russia. In each study, a prefieldwork focus group was held with outreach workers from the local harm reduction NGOs to obtain information about the drug scene and to identify seeds to begin recruitment. Respondents received the same primary incentives for participating in the RDS study as in the IFS study and also an additional secondary incentive for each respondent they recruited into the study. ${ }^{22,24}$

In all studies FWs recorded their observations on the drug scene, progress of the fieldwork and any difficulties arising from the research in detailed notes. These observations provide a useful additional comparison between the two sampling methods.

\section{Duration and Cost of Fieldwork}

We compared the duration of fieldwork for the IFS and RDS methods by calculating the mean number of days of fieldwork for each method and the proportion of the sample recruited on each day as the studies progressed. Means were compared using $t$-tests.

Costs were estimated for five of the seven surveys conducted in Russia and analysis focused on examining the cost effectiveness of recruiting a given sample for each of the sampling methodologies from a programmatic point of view as opposed to examining societal or health system costs. The IFS studies in Moscow, Barnaul and Volgograd were conducted in 2003 and the RDS studies in Togliatti, Barnaul and Volgograd were conducted in 2004. Costs were calculated as: (1) 'outside' costs including salary, accommodation and travel of field work consultants; (2) local salary costs of FWs and researchers; (3) recruitment costs including the packages of goods valued at 140 roubles and 300 roubles, respectively, for primary and secondary incentives; and (4) other costs including local transport, telephone calls and logistical costs of training FWs. For the RDS study the cost of the fixed site used for interviews is not included as an explicit cost, rather it is subsumed into the local salary costs since local staff contracted to undertake the work were employed from syringe exchange programmes. Costs are presented assuming that there are elements of fixed and variable costs at each sample size and that an extra 20 respondents will require keeping the entire survey team in the field for one extra day. 


\section{Demographic and Injecting Risk Behaviours of Sample}

Demographic and injecting risk behaviours of IDUs recruited through IFS and RDS were compared in the two sites (Volgograd and Barnaul) where both survey methodologies were used to ensure a cleaner comparison between survey methods. Demographic and injecting characteristics were used as the outcome variables with recruitment method included as an independent variable. In the univariate analysis, chi-squared tests were used to compare outcomes for categorical variables and Bartlett's test for equal variance to compare continuous variables. For the multivariate analysis, logistic regression models were used to explore associations between explanatory variables and a binary outcome, multinomial logit models were used for categorical variables with multiple values and ordinary least squares for continuous variables. The multivariate analysis includes all common independent variables and a categorical variable indicating survey method used. This allows outcomes to be compared controlling for all independent variables and to identify impacts associated with only survey methodology. All statistical analyses were conducted using Stata 7 with significance set at 5\% (Stata Corporation, College Station, Texas).

\section{RESULTS}

\section{The Surveys}

A total of 3,771 IDUs were recruited into nine surveys across four cities in the Russian Federation and two cities in Estonia (Table 1). A total of 2,049 (54\%) participants were recruited through IFS and 1,722 (46\%) through RDS. Only IDUs are included in the analyses we present here.

\section{Duration of Field Work}

The mean (standard deviation) duration of fieldwork for IFS surveys was 23.8 (4.1) days and for RDS $20.6(0.9)$ days $(t=27.9, \mathrm{p}<0.001)$. Figure 1 depicts the number of respondents recruited by each successive day of fieldwork by city and recruitment method. The RDS studies appear to follow a pattern of recruitment that we might expect: the number of respondents increases steadily as the number of waves increase and then declines towards the end of the study as completion of the target sample size approaches and respondents are asked to refer fewer contacts to the study. Kohtla Jarve, Estonia, appears to be an exception to this as recruitment peaks more sharply then abruptly finishes. The recruitment for the IFS studies does not appear to follow any set pattern across the cities. In Moscow the highest number of respondents in any 1 day occurs at the start of the study. In Barnaul, and to a less extent Volgograd, the number of participants recruited per day is more even across the duration of the study.

\section{Sample Characteristics}

Table 2 summarizes the characteristics of IDUs by recruitment method from the four surveys conducted in Volgograd and Barnaul. In both cities, RDS participants were younger, more likely to be male, to have attended higher education and to have official residency permits for the city. RDS participants were more likely to report injecting heroin in both sites and less likely to report injecting vint or mak*

\footnotetext{
"Vint is a liquid metamphetamine and mak is a liquid opiate derived from poppy straw.
} 


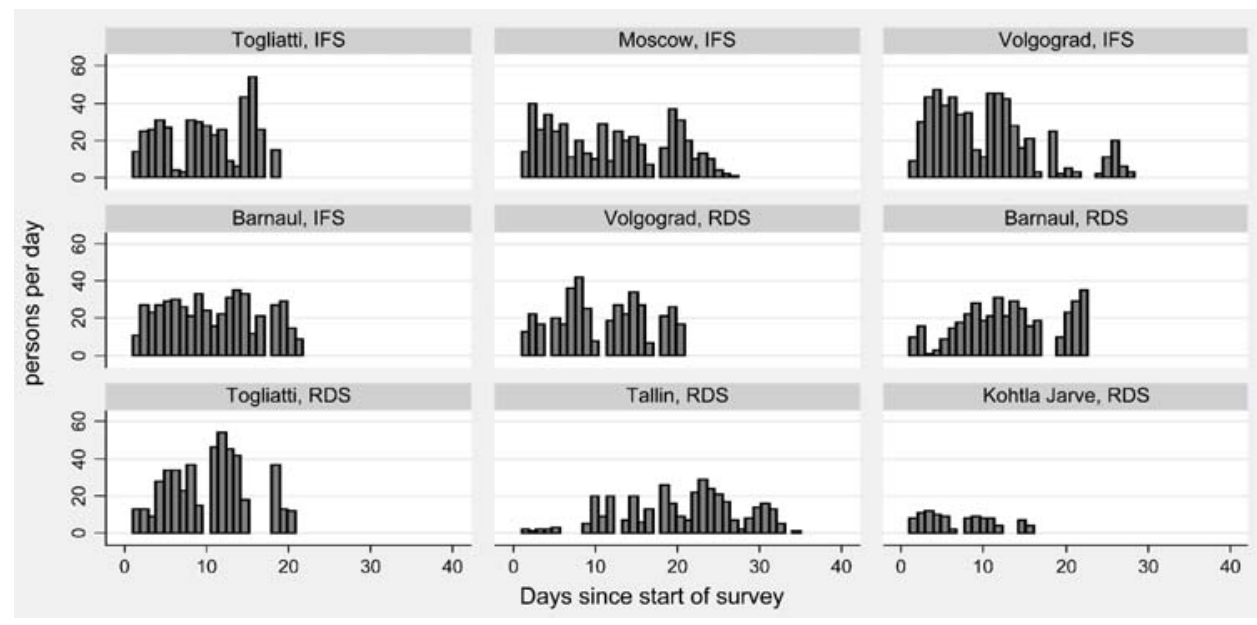

FIGURE 1. Frequency of recruitment per day amongst studies of injecting drug users in Russia and Estonia (2001-2005), by city and recruitment method.

than IFS participants. Frequency of injecting did not differ by recruitment method in either city. Regarding injecting risk behaviour, there was no difference between recruitment methods in the proportion of IDUs reporting injecting with a used needle/syringe in the last 4 weeks in Volgograd but in Barnaul a higher proportion of RDS respondents reported this behaviour ( 21 vs. $15 \%, \mathrm{p}=0.02$ ). In Volgograd IFS respondents were more likely to report ever having injected with a used needle/ syringe than RDS respondents ( 61 vs. $40 \%, \mathrm{p}<0.001$ ). The opposite was found in Barnaul (53 vs. 63\%, respectively, $\mathrm{p}<0.003$ ). In both cities and with both methods, the main source of new needles/syringes was pharmacies. A higher proportion of IFS respondents reported using needle/syringe exchanges or treatment centres in both cities and in Barnaul a higher proportion of RDS respondents reported using a source other than needle/syringe exchange (defined as friend, dealer, family or on the street) as their main source of needles/syringes.

\section{Multivariate Analysis}

Table 3 summarizes the multivariate analysis for the categorical and continuous variables for Barnaul and Volgograd. After controlling for all independent variables, our findings indicate that RDS recruited a population 0.07 years younger in both cities. In both cities RDS participants had been injecting slightly longer than IFS participants. In Barnaul, RDS participants were less likely to report obtaining their new needles/syringes from pharmacies $(-5 \%)$ but there was no evidence to suggest a difference in Volgograd. In Volgograd RDS participants were less likely to report using needle/syringe exchanges $(-3 \%)$ but there was no difference in Barnaul. RDS participants in Barnaul were $4 \%$ more likely to report using another source for their new needles/syringes (Table 3).

In the logistic regression analysis (Table 4), RDS was more likely to result in a higher proportion of male IDUs (Barnaul OR $=2.0$, Volgograd OR $=3.8$ ) and participants who had attended higher education (Barnaul OR $=5.2$, Volgograd $\mathrm{OR}=3.0)$. In Barnaul RDS participants were more likely to have official residency permits $(\mathrm{OR}=4.6)$ but not in Volgograd. In both cities, RDS participants were more likely to inject heroin over mak or vint than IFS participants (Barnaul OR $=2.5$ and 


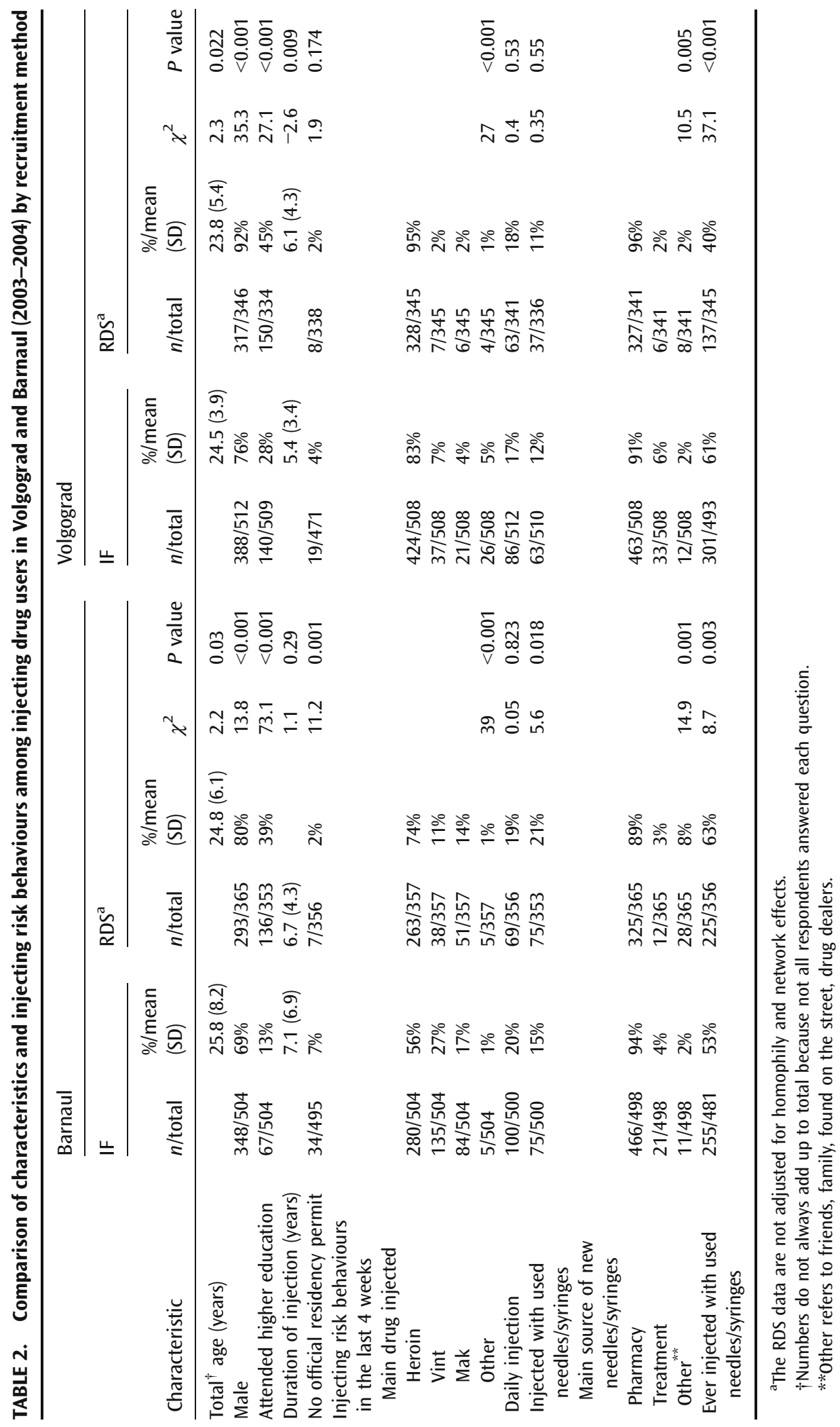




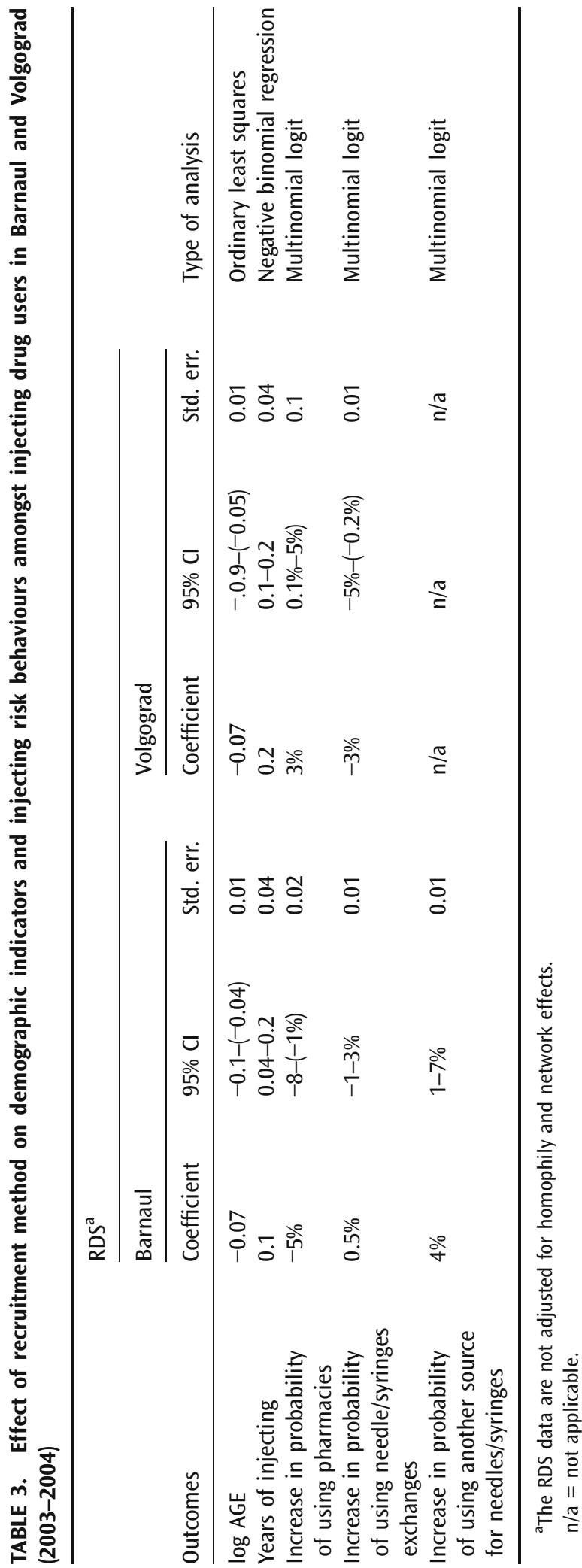




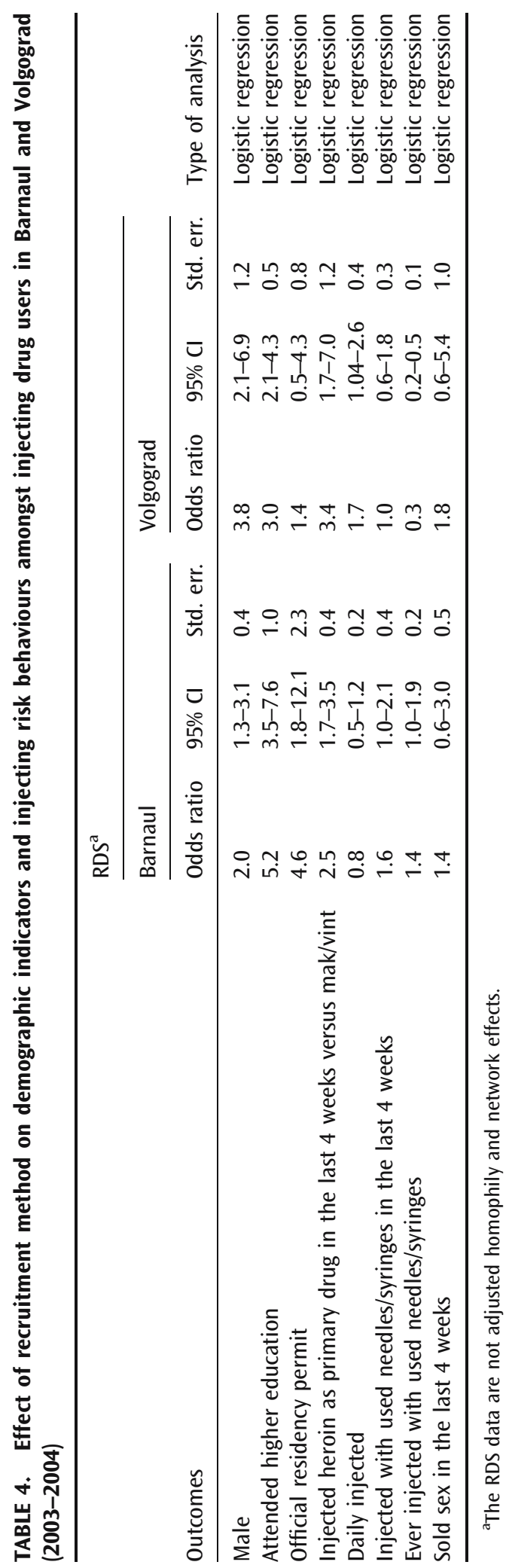


Volgograd OR = 3.4). In Volgograd RDS participants had almost twice the odds of reporting daily injection $(\mathrm{OR}=1.7)$ than IFS participants and had reduced odds of ever injecting with used needles/syringes $(\mathrm{OR}=0.3)$. In Barnaul, RDS participants were more likely to report injecting with used needles/syringes in the last 4 weeks and ever $(\mathrm{OR}=1.6$ and $\mathrm{OR}=1.4$, respectively $)$.

\section{Costs}

The total cost of conducting an IFS survey recruiting 400 respondents was estimated to be $\$ 14,651$ (USD) but $\$ 16,100$ for the RDS survey (Table 5). This translates to $\$ 43$ per respondent using RDS and $\$ 37$ using the IFS method. Increasing the sample from 400 to 500 reduced the average cost per respondent by $\$ 1$ for the RDS method and by $\$ 3$ for the IFS method. Reducing the sample from 400 to 300 respondents increased the cost per respondent by $\$ 2$ for the RDS method and $\$ 5$ for the IFS method. These results are presented in Table 5.

\section{DISCUSSION}

Our findings suggest that RDS does appear to be a faster recruitment method and that there are significant differences in the demographic characteristics of IDUs recruited via RDS in comparison with those recruited via IFS. However, evidence from the two cities is conflicting with regard to whether RDS recruits IDUs who engage in riskier injecting practices.

One of the suggested benefits of RDS is its apparent ability to recruit the hardest to reach sections of hidden populations. ${ }^{22}$ We found some differences in measures of marginalization and risk behaviours between the two recruitment methods, RDS participants tend to be slightly younger and are less likely to use

TABLE 5. Analysis of costs of recruiting IDUs and sex workers from five surveys in Moscow, Volgograd and Barnaul, Russian Federation (2003-2004) by recruitment method and sample size

\begin{tabular}{|c|c|c|c|c|c|c|}
\hline \multirow[b]{3}{*}{ Sample size } & \multicolumn{6}{|c|}{ Recruitment method } \\
\hline & \multicolumn{3}{|c|}{ Indigenous field workers } & \multicolumn{3}{|c|}{ Respondent-driven sampling } \\
\hline & 300 & 400 & 500 & 300 & 400 & 500 \\
\hline Outside $\operatorname{costs}^{\mathrm{a}}$ & -15.40 & $\$ 20.30$ & 11.40 & -0.18 & $\$ 19.62$ & 0.18 \\
\hline Local costs ${ }^{b}$ & -13.20 & $\$ 7.93$ & 10.40 & -0.25 & $\$ 5.50$ & 0.25 \\
\hline Recruitment of respondents ${ }^{c}$ & -33.30 & $\$ 7.00$ & 20.00 & -0.25 & $\$ 9.95$ & 0.25 \\
\hline Other & 0.00 & $\$ 1.40$ & 0.00 & -0.25 & $\$ 5.25$ & 0.25 \\
\hline Total & -17.20 & $\$ 14,651.00$ & 12.60 & -0.21 & $\$ 16,100$ & 0.21 \\
\hline Cost per respondent & $\$ 42.00$ & $\$ 37.00$ & $\$ 34.00$ & $\$ 43.00$ & $\$ 41.00$ & $\$ 40.00$ \\
\hline
\end{tabular}

At the time of writing 28 rubles was equivalent to one US Dollar. The 2003 costs are adjusted for inflation that occurred between August 2003 and 2004 based on price indices taken from the Bank of Russia.

${ }^{a}$ Costs are presented assuming that there are elements of fixed and variable costs in each sample size and that an extra 20 respondents will take one extra day necessitating employing the entire fieldwork team for that extra day.

${ }^{\mathrm{b}}$ In order to protect the confidentiality of staff we report only the total amount of all salaries and fees paid to project staff.

'The costs for the RDS surveys are also based on providing a package of goods valued at 140 rubles for each survey participant and a secondary reward of 300 rubles for each participant recruited. Costs for the IFS surveys are based on providing a package of goods valued 140 rubles for each participant and no secondary reward. 
needle/syringe exchange programs. Some evidence in Russia suggests that IDUs whose primary source of needles/syringes is informal are at more risk of engaging in high-risk behaviours. ${ }^{31}$ However, IFS participants were less likely to have attended higher education and have official residency permits to live in the city and more likely to be female. Lack of residency permit is an indicator of marginalization as it will affect an individual's ability to use health services or obtain employment. ${ }^{32,33}$

As no consistent trend emerges from the analysis of the effect of recruitment method on sample characteristics, then choice of method might be made on the basis of methodology and cost.

\section{Inclusion Criteria and Data Validity}

With IFS the responsibility for selecting the right target group is placed with the FWs, and its success depends on establishing a trusting relationship between the researchers and the fieldwork team. With RDS, issues of trust are less important, as researchers undertake the interviews themselves. The problems of establishing whether respondents are genuine members of the target group remain. Although measures can be put in place which might reduce this from happening, such as using indigenous field workers to screen participants or recording biometric measurements to avoid the same respondent being interviewed twice, it is very difficult to measure to what extent fabricated data may enter a survey.

A disadvantage of both methods is that study participants who are not members of the target group may lie about their membership in order to receive a reward. This was the case in two of the IFS sites where 9 and $14 \%$ of questionnaires were subsequently found to be fakes. This was discovered because strict validation processes had been set up and there was a good relationship between the FW supervisor and indigenous field workers. In the Togliatti RDS study 15 people were refused entry into the study, as they were suspected of not being current injectors. However these may be considered a minimum estimate as one cannot rule out that additional fabrication might have occurred and gone undetected. Having a modest primary and secondary incentive can minimize the chances that participants who are not members of the target population will be recruited.

Determining the best incentive size is difficult and has many implications for the study, especially for RDS studies where the secondary incentive is so crucial to recruitment success. The networks recruited through RDS are largely artificial, created as a result of the study and since their composition is dependent on the incentive, changes to the amount of incentive offered would change the composition of the network. This is illustrated with the case of sex workers in Eastern Europe, who have been found to be harder to recruit through RDS in part due to the small incentive and social network properties; this is discussed in more detail in a paper in this issue by Simic et al.

Adjusting the RDS sample to obtain 'population' estimates depends on the ability to recruit a random population within a subject's social networks and a positive probability of recruiting everyone in that network. The possibility that the network is highly dependant on the incentive raises the question whether the latter condition obtains. This is particularly relevant when the definition of the population of study is fluid or artificially constructed by the research as with IDUs and sex workers. It should also be noted that the collection of information describing network characteristics which allows RDS analysis to produce 'population' estimates requires the respondent to recall detailed information on 
the composition of their network, including its size and each member's relationship with the recruiter. This process carries a large potential for error.

\section{Personal Safety and Capacity Building}

There are safety considerations that favour RDS as respondents attend a fixed site for an interview in which a minimum number of staff is always present. In the IFS method, interviewers may find themselves travelling to an area in which they are unfamiliar, and unintentionally put themselves in danger, especially if it becomes known that they are carrying financial rewards or gift packs.

\section{LIMITATIONS}

Whilst we have tried to limit confounding in our analysis by comparing RDS and IFS studies conducted in the same cities, the studies were conducted in different years, and the findings may be confounded by time. Time may be important in relation to behaviour, but is likely to be less important in relation to socio demographic characteristics of the target group. None of the studies were set up specifically with the aim of comparing sampling methodologies, and this limited the number of characteristics that could be compared between study methodologies. A study set up specifically with the aim of comparing the methodologies might produce different results and facilitate more detailed comparison of characteristics. Additionally the starting point for both the IFS and RDS studies in all sites was the local outreach team. This may have led to more similarities in the sample composition than would have occurred if seeds had been selected through other methods. However, according to the principles of RDS, the selection of seeds does not ultimately influence the composition of the sample, since after several waves of recruitment the sample should be independent of the non-randomly selected seeds. $^{24}$

\section{CONCLUSION}

The HIV epidemic is driven by populations engaging in high-risk behaviours mixing with those engaging in lower risk behaviours. It is important to identify the parameters of risk behaviour in order to model these epidemics and to design appropriate interventions. If we assume that, after adjusting for network sizes and homophily, RDS is more successful at estimating risk behaviours across a more representative population than IFS, then it could lead to more effective modelling and prediction of such epidemics; however to date there is no evidence to suggest that this is the case. Our findings indicate that as a recruitment strategy, RDS is no better than IFS in identifying populations with highest risk behaviours. It does have practical advantages in terms of safety of the FW team, with faster recruitment at only additional costs. In the meantime, until the statistical superiority of RDS can be proven, a preferred approach may be to adopt the best aspects of both methodologies, depending on the resources available. A combination could include the use of coupons for recruitment, but also training indigenous field workers to work alongside researchers to undertake interviews, serving to increase their capacity in research skills whilst ensuring that the correct target group is being reached. 


\section{CONFLICTS OF INTEREST STATEMENT:}

None to declare.

\section{REFERENCES}

1. Hamers FF, Downs AM. HIV in central and eastern Europe (review). Lancet. 2003; 361(9362): 1035-1044.

2. Rhodes T, Ball A, Stimson G, et al. HIV infection associated with drug injecting in the Newly Independent States, eastern Europe: the social and economic context of epidemics. Addiction. 1999;94:1323-1336.

3. Rhodes T, Sarang A, Bobrik A, Bobkov E, Platt L. HIV transmission and HIV prevention associated with injecting drug use in the Russian Federation. Int J Drug Policy. 2004; 15:39-54.

4. AFEW. Officially registered HIV cases in the Russian Federation, 1 January 1987 to 31st December, 2005. Available at: http://www.afew.org/english/statistics/HIVdata-RF.htm. 2005.

5. UNAIDS/WHO. Guidelines for second generation HIV surveillance: the next decade. Geneva, Switzerland: World Health Organization; 2000, p. 48.

6. Bishop YMM, Fienberg SE, Holland PW. Estimating the Size of a Closed Population. Discrete Multivariate Analysis: Theory and Practice. Cambridge, Massachusetts: MIT; 1975.

7. EMCDDA. Methodological Guidelines to Estimate the Prevalence of Problem Drug Use at Local Level. Lisbon: European Monitoring Centre for Drugs and Drug Addiction; 2000.

8. UNDCP. GAP Toolkit Module 3: Estimating Prevalence-Indirect Methods for Estimating the Size of the Drug Problem. Vienna: International Centre Austria; 2002.

9. Diaz T, Des Jarlais DC, Vlahov D, et al. Factors associated with prevalent hepatitis C: differences among young adult injection drug users in lower and upper Manhattan, New York City. Am J Public Health. 2001;91(1):23-30.

10. Griffiths P, Gossop M, Powis B, Strang J. Researching hidden populations of drug users by privileged access interviewers: methodological and practical issues. Addiction. 1993;88:1617-1626.

11. Kuebler D, Hausser D, Gervasoni J-P. The characteristics of 'new users' of cocaine and heroin unknown to treatment agencies: results from the Swiss Hidden Population Study. Addiction. 2000;95(10):1561-1571.

12. Watters JK, Biernacki P. Targeted sampling: options for the study of hidden populations. Soc Probl. 1989;46:416-430.

13. Rhodes T, Platt L, Maximova S, et al. Prevalence of HIV, hepatitis $\mathrm{C}$ and syphilis among injecting drug users in Russia: multi-city study. Addiction. 2006;101(2):252-266.

14. Goodman LA. Snowball sampling. Ann Math Stat. 1961;32:148-170.

15. Broadhead RS, Heckathorn DD, Grund J-P, Stern LS, Anthony DL. Drug users versus outreach workers in combating AIDS: preliminary evidence of a peer-driven intervention. J Drug Issues. 1995;23:531-564.

16. Albery I, Strang J, Gossop M, Griffiths P. Illicit drugs and driving: prevalence, beliefs and accident involvement among a cohort of current out-of-treatment drug users. Drug Alcohol Depend. 2000;58(1-2):197-204.

17. Des Jarlais DC, Johnston P, Friedmann P, et al. Patterns of HIV prevalence among injecting drug users in the cross-border area of Lang Son Province, Vietnam, and Ning Ming County, Guangxi Province, China. BMC Public Health. 2005;5(89):1-8.

18. Hunter GM, Stimson G, Judd A, Jones S, Hickman M. Measuring injecting risk behaviour in the second decade of harm reduction: a survey of injecting drug users in England. Addiction. 2000;95(9):1351-1361. 
19. Judd A, Hickman M, Jones S, et al. Incidence of hepatitis $C$ virus and HIV among new injecting drug users in London: prospective cohort study. BMJ. 2005;330(7481):24-25.

20. Robson P, Bruce M. A comparison of 'visible' and 'invisible' users of amphetamine, cocaine and heroin: two distinct populations? Addiction. 1997;92(12):1729-1736.

21. Stimson GV, Jones S, Sullivan D, Chalmers C. A short questionnaire (IRQ) to assess injecting risk behaviour. Addiction. 1998;93(3):337-347.

22. Heckathorn DD. Respondent-driven sampling: a new approach to the study of hidden populations. Soc Probl. 1997;44(2):174-198.

23. Kilworth PD, Johnsen EC, Bernard HR, Shelly GA, McCarthy C. The reversal small world experiment. Soc Netw. 1978-79;1:159-192.

24. Heckathorn DD. Respondent-driven sampling. II. Deriving valid population estimates from chain-referral samples of hidden populations. Soc Probl. 2002;49(1):11-34.

25. Salganik MJ, Heckathorn DD. Sampling and estimation in hidden populations using respondent-driven sampling. Sociol Method. 2004;34:193-239.

26. Power R. Some methodological and practical implications of employing drug users as indigenous field workers. In: Boulton M, ed. Challenge and Innovation: Methodological Advances in Social Research on HIVIAIDS. London: Taylor and Francis; 1994:97-111.

27. Magnani R, Sabin K, Saidel T, Heckathorn DD. Review of sampling hard-to-reach and hidden populations for HIV surveillance. AIDS. 2005;19(Suppl 2):S67-S72.

28. Platt L, Bobrova N, Rhodes T, et al. High HIV prevalence among injecting drug users in Estonia: implications for understanding the risk environment. AIDS. 2006 (in press).

29. Rhodes T, Lowndes CM, Judd A, et al. Explosive spread and high prevalence of HIV infection among injecting drug users in Togliatti City, Russia. AIDS. 2002;16:F25-F31 (May 29).

30. Wall M. Sex and drugs and economic behaviour: results from a survey of drug injectors and sex workers in two Russian cities. 2006 (in press).

31. Rhodes T, Judd A, Mikhailova L, et al. Injecting equipment sharing among injecting drug users in Togliatti City, Russian Federation. J Acquir Immune Defic Syndr. 2004;35(3):293-300.

32. Konings E. Prostitution and HIV/AIDS in CEE/CIS. Geneva, Switzerland: UNAIDS; 1996.

33. Lakhulamani V. The prostitution situation in a number of cities of Russia, Ukraine and Byelarus. Zhurnal mikrobiologii, epidemiologii, i immunobiologii. 1997;1:102-104 (Jan-Feb).

34. Platt L, Rhodes T, Sarang A, Milhailova L. Ongoing HIV epidemic amongst injecting drug users in Togliatti City, Russian Federation. London School of Hygiene and Tropical Medicine. Unpublished, 2006. 